\title{
Factors Associated with Maternal and Child Health Services Uptake and Their Association with Health Outcomes in Mashonaland East, Zimbabwe
}

\author{
Maxwell Mhlanga ${ }^{1 *}$, Zvinavashe $\mathbf{M}^{2}$, Gwanzura $\mathbf{L}^{2}$ and Babill Stray-Pedersen ${ }^{3}$ \\ ${ }^{1}$ Faculty of Health Sciences, University of Zimbabwe, Harare, Zimbabwe \\ ${ }^{2}$ College of Health Sciences, University of Zimbabwe, Harare, Zimbabwe \\ ${ }^{3}$ University of Oslo, Norway
}

\begin{abstract}
This study aimed at determining the factors associated with maternal and child health services uptake and their association with maternal and child health outcomes.

Design and setting: The study was conducted in two districts in Mashonaland East namely Murewa and Seke in Zimbabwe. An analytical cross sectional study design was conducted between November 2016 and March 2017. Women with children 0-48 months who were being recruited for an interventional study were targeted with an aim of determining the baseline characteristics and comparability of participants in the intervention and control arm. A sample of 672 mothers was interviewed. Data was analyzed in SPSS version 20 and STATA 13.

Main outcome(s): The study focused on maternal and child health outcomes.

Results: The mean age for the women was 28.0 years $(S D=6.8)$ and the mean birth weight for children was 3061 $\mathrm{g}(\mathrm{SD}=537)$. Women had a mean weight of $62.5 \mathrm{~kg}(\mathrm{SD}=11.5)$ and the mean number of children per woman were 2.6 $(S D=1.5)$. In this study $154(22.9 \%)$ of the participants booked for Antenatal Care (ANC) in the first trimester and 321 $(47.7 \%)$ and $171(25.4 \%)$ booking in the second and third trimester respectively. There was a statistically significant association between a child's gestational age and birth weight (OR=2.14; 95\% Cl: 1.22-3.75). Maternal complications were significantly associated with the number of children delivered prior to the last pregnancy $(\mathrm{OR}=4.4 ; 95 \% \mathrm{Cl}: 2.45-$ 8.04). First ANC timing was strongly associated with the place of delivery (OR=2.84, $95 \% \mathrm{Cl}$ : $1.53-5.25)$ and so was ANC registration decision making $(\mathrm{OR}=3.52 ; 95 \% \mathrm{Cl}: 1.88-6.58)$. The weaning time was significantly associated with child morbidity $(\mathrm{OR}=5.28 ; 95 \% \mathrm{Cl}: 2.57-9.86)$

Conclusion: This study revealed that though there is a satisfactory health seeking behavior among pregnant and lactating women, there is still a significant gap in knowledge of critical recommended Maternal Neonatal and Child Health $(\mathrm{MNCH})$ practices. Good knowledge and practices are essential in the reduction of preventable maternal and child morbidity and mortality. Universal maternal health access is only achievable if the women and the community take decisions about their own health in a supportive environment; hence the need to have community based interventions for maternal health access. The results of the study have shown that the quality and methods of delivery of antenatal care education need to reviewed to improve effectiveness of antenatal care.
\end{abstract}

Keywords: Maternal morbidity; Utilization of maternal services; Health outcomes

\section{Introduction}

Consistent access and utilization of $\mathrm{MNCH}$ services has been proven to reduce mortality and morbidity. Utilization of maternal and child health services is a function of one's health care seeking behavior and this is influenced by a lot of factors. Indicators for uptake of $\mathrm{MNCH}$ services include: ANC attendance, family planning, use of ANC services such as screening tests, physical examination, and prophylactic treatment for conditions like tetanus, anemia and malaria. Other indicators include, delivering at a health facility and receiving postnatal care (PNC) services in line with the recommended guidelines. This also includes child care indicators such as child immunizations, prevention and management of child of childhood illnesses.

The World Health Organization (WHO) came up with the 3 delay model to determine where problems with access and utilization of services are concentrated for policy formulation. The three delays are delay in deciding to seek care, delay in reaching the health facility and delay in receiving appropriate care at the health facility [1]. It can be deduced from the way the delays are structured that the third delay is heavily influenced by the other two delays. In Zimbabwe, survey reports have approximated that about $70 \%$ of the causes of maternal mortality and morbidity emanate from the first and second delay with the first delay carrying the greater proportion [2]. However, little is known about the key factors that result in the delays at community and household level and nothing much has been said about the strength of each predictor in determining the likelihood of mortality and morbidity. On the same note, the thrust on health systems strengthening in Zimbabwe has been on health facility systems and nothing significant has been done to strengthen the community health system delivery.

A cross sectional study using mixed methods was conducted in Eastern Ethiopia with 561 women of reproductive age who gave birth in the last 2 years [1]. Bivariate and multivariate logistic regressions

${ }^{*}$ Corresponding author: Maxwell Mhlanga, Faculty of Health Sciences, University of Zimbabwe, Harare, Zimbabwe, Tel: +263773199626; E-mail: profmaxmhlanga7@gmail.com

Received August 04, 2017; Accepted September 11, 2017; Published September 13, 2017

Citation: Mhlanga M, Zvinavashe M, Gwanzura L, Stray-Pedersen B (2017) Factors Associated with Maternal and Child Health Services Uptake and Their Association with Health Outcomes in Mashonaland East, Zimbabwe. Clinics Mother Child Health 14: 272. doi: 10.4172/2090-7214.1000272

Copyright: $\odot 2017$ Mhlanga M, et al. This is an open-access article distributed under the terms of the Creative Commons Attribution License, which permits unrestricted use, distribution, and reproduction in any medium, provided the original author and source are credited. 
Citation: Mhlanga M, Zvinavashe M, Gwanzura L, Stray-Pedersen B (2017) Factors Associated with Maternal and Child Health Services Uptake and Their Association with Health Outcomes in Mashonaland East, Zimbabwe. Clinics Mother Child Health 14: 272. doi: 10.4172/20907214.1000272

Page 2 of 7

were employed to identify associated factors and odds ratios were used to measure the strength of association. The study concluded that the health seeking behavior of women was low and mainly influenced by education level, knowledge on complications and birth order. Focused health education with kind and supportive health care provider counseling was recommended for the improvement of health care seeking behaviors of women.

A cross sectional survey was conducted in Nigeria on socio-demographic factors and appropriate health-care seeking behaviors for childhood illnesses. High maternal education and early care seeking, utilization of orthodox health facilities and drug use at home age $<1$ year were associated with early care seeking outside home and utilization of orthodox health services $(\mathrm{p}<0.001)$ [3]. Logistic regression showed that high maternal education and high family socio-economic status were strong predictors of early care seeking and care seeking outside home, maternal age, maternal education and family socio-economic status are predictors of appropriate health care-seeking behaviors for children [3].

This study aimed at assessing the current status quo in Mashonaland East province with regards to selected key MNCH indicators. This survey was done to assess the health seeking behaviors of mothers in Mashonaland East and its association with maternal and child health outcomes. This will then be followed by a cluster randomized control trial that will employ a developed community mobilization approach, comparing its effectiveness in improving health outcomes with the current conventional community mobilization approach.

\section{Materials and Methods}

This was an analytical cross sectional study. The study was conducted in two districts of Mashonaland East province of Zimbabwe, namely Murehwa and Seke district. Participants were drawn from the motherchild pairs who were being recruited for a cluster randomized controlled trial and the study sample comprised of 672 mothers who had children 0-48 months. The Cluster Randomized Control trial sample size was calculated in STATA 13 software, assuming morbidity prevalence of 50\% with a margin error of $5 \%$. The assumed intra-cluster correlation coefficient (ICC) was 0.05 with a level of significance of $5 \%$; design effect of 2.45 and power 0.9 . Firstly, assuming individual randomization the sample size per arm would have been 121 subjects. Allowing for cluster randomization, the sample size per arm became 330 mother-child pairs and the average number of clusters per arm $(\mathrm{m})$ was 11 as determined from the software. All of the 672 mothers were interviewed. All women with children 0-48 months who were coming to the 4 respective health centers sampled for the study were eligible and hence included in the study. The study excluded women who were mentally ill and those who were seriously ill or unconscious. Four research assistants who by qualification were all trained mid-wives received training on data collection and each collected data at one of the 4 health facilities. Interviews were conducted in private rooms at the health facility. Data was analysed in Statistical Package for Social Science (SPSS version 20.0) and Stata 13. Both descriptive and inferential statistics were used in data analysis.

The study was approved by the Joint Research Ethics Council for Parirenyatwa and the University of Zimbabwe (JREC), The Medical Research Council of Zimbabwe (MRCZ) and the Provincial Medical Director of Mashonaland East. Written informed consent was sought and provided by all the 672 participants and participants were notified that they can terminate the interview at any given moment without questions or any negative consequences. Data storage was done in a manner to assure confidentiality and privacy. Study- related documents were kept under lock and key.

\section{Results}

\section{Sociodemographic data}

The study comprised of women of child bearing age who had children 0-48 months drawn from two districts in Mashonaland East namely Murehwa and Seke district. The participating health centres were Murewa polyclinic and Macheke clinic in Murewa district; Kunaka rural Hospital and Marirangwe clinic in Seke district. The mean age for the women was 28.0 years $(\mathrm{SD}=6.8)$ and the mean birth weight for children was $3061 \mathrm{~g}(\mathrm{SD}=537)$. Women had a mean weight of 62.5 $\mathrm{kg}(\mathrm{SD}=11.5)$ and the mean number of children per woman were 2.6 $(\mathrm{SD}=1.5)$. Of the 672 participants interviewed, 568 (84.5\%) said their households were head by men. Thirty percent (202) of the participants had not reached the ordinary level of education ('O' level) and in terms of marital status $579(86.2 \%)$ were married. Table 1 below summarises the socio-demographic characteristics for the study.

The study assessed the levels of utilization of $\mathrm{MNCH}$ services amongst women of child bearing age. The study ascertained the prevalence of utilization of health services during pregnancy; delivery and post-natal phase. Timing for the first ANC visit and total number of ANC visits attended prior to delivery were also checked. Overall, 639 (95.2\%) of the participants reported having received health services at least once during their last pregnancy, 599 (89.1\%) during delivery and $66(94.2 \%)$ post-delivery. Reasons for not accessing health care services along the continuum of care were explored. Religious beliefs were cited as the reason for not accessing health care services by $10(23.2 \%)$ of those who did not seek care during pregnancy. Of the 43 who did not access any service during pregnancy, $6(14 \%)$ said the health center is too far and $9(20.9 \%)$ cited financial constraints for user fees. Reasons cited for failure to access services during delivery included distance to the clinic 26 (60.5\%), resulting in emergencies before arrival; religious beliefs and financial constraints. Table 2 below summarizes the results on access and utilization of $\mathrm{MNCH}$ services.

\section{Complications experienced along the continuum of care}

The study also determined the prevalence of maternal morbidity. This includes prevalence of maternal complications and co-morbidities along the continuum of care. The study revealed that $4.9 \%$ of the 672 participants had confirmed proteinuria and the prevalence was comparable in both the control and intervention arm participants, $17.9 \%$ and $19.6 \%$ respectively for those participants who were screened for proteinuria. Of the 672 participants interviewed, $13.2 \%$ were HIV positive and receiving treatment from their respective health centers. About $94 \%$ of the participants had a normal delivery in their last pregnancy and only 68 (10.1\%) of the participants reported ever having a complication during pregnancy. Of the 68 participants who had complications during pregnancy; 32 (47.1\%) had-pre-eclampsia; 12 (17.6\%) had anemia; 11 (16.2\%) antepartum hemorrhage; 63 (92.6\%) had severe headache. Nine percent of the participants had complications during delivery and of these 20 (66.7\%) had eclampsia; 13 (21.7\%) had severe hemorrhage; 20 (66.7\%) had obstructed labor and $1(1.7 \%)$ had a retained placenta. In the postpartum period, 68 (10.1\%) had complications. Of these, 34 (50\%) had post-partum hemorrhage; 4 (5.9\%) were depressed; 2 (2.9\%) had sepsis; 9 (13.2\%) had high blood pressure and 3 (4.4\%) were severely weak.

\section{Knowledge on MNCH}

In this study the participants' knowledge levels on selected $\mathrm{MNCH}$ issues was assessed. Mothers were interviewed on knowledge with regards to the benefits of ANC both to the mother and the unborn baby, knowledge of at least 2 danger signs along the continuum of care (preg- 
Citation: Mhlanga M, Zvinavashe M, Gwanzura L, Stray-Pedersen B (2017) Factors Associated with Maternal and Child Health Services Uptake and Their Association with Health Outcomes in Mashonaland East, Zimbabwe. Clinics Mother Child Health 14: 272. doi: 10.4172/20907214.1000272

Page 3 of 7

\begin{tabular}{|c|c|c|c|c|c|c|c|}
\hline \multicolumn{4}{|l|}{ Murewa District } & \multicolumn{4}{|c|}{ Seke District } \\
\hline & Macheke & Murewa & Sub-total & Marirangwe & Kunaka & Sub-total & Overall Proportion \\
\hline & $n=125$ & $n=254$ & $n=379$ & $n=112$ & $n=181$ & $n=293$ & $\mathrm{~N}=672$ \\
\hline \multicolumn{8}{|l|}{ Variable } \\
\hline $\begin{array}{l}\text { Sex of Household Head }(\mathrm{HH}) \\
\text { Males } \\
\text { Females }\end{array}$ & $\begin{array}{c}109(87.2 \%) \\
16(12.8 \%)\end{array}$ & $\begin{array}{c}213(83.9 \%) \\
41(16.1 \%)\end{array}$ & $\begin{array}{c}422(85.0 \%) \\
57(15 \%)\end{array}$ & $\begin{array}{l}95(85.8 \%) \\
17(14.2 \%)\end{array}$ & $\begin{array}{c}159(87.8 \%) \\
22(12.2 \%)\end{array}$ & $\begin{array}{c}259(88.4 \%) \\
29(11.6 \%)\end{array}$ & $\begin{array}{l}568(84.5 \%) \\
104(15.5 \%)\end{array}$ \\
\hline $\begin{array}{l}\text { No. of Children per } \mathrm{HH} \\
<4 \\
4 \text { and above }\end{array}$ & $\begin{array}{l}71(56.8 \%) \\
32(43.2 \%)\end{array}$ & $\begin{array}{c}144(56.7 \% \\
155(43.3 \%)\end{array}$ & $\begin{array}{c}215(56.7 \%) \\
32(43.3 \%)\end{array}$ & $\begin{array}{l}85(75.9 \%) \\
27(24.1 \%)\end{array}$ & $\begin{array}{c}138(76.2 \%) \\
42(23.8 \%)\end{array}$ & $\begin{array}{c}223(76.1 \%) \\
32(23.9 \%)\end{array}$ & $\begin{array}{l}437(65.2 \%) \\
235(34.8 \%)\end{array}$ \\
\hline $\begin{array}{l}\text { Literacy of respondents } \\
\text { <" O" level } \\
\text { "O" \& above }\end{array}$ & $\begin{array}{l}51(40.8 \%) \\
74(59.2 \%)\end{array}$ & $\begin{array}{c}73(28.7 \%) \\
181(71.3 \%)\end{array}$ & $\begin{array}{l}124(32.7 \%) \\
255(67.3 \%)\end{array}$ & $\begin{array}{l}41(36.6 \%) \\
70(63.4 \%)\end{array}$ & $\begin{array}{c}23(12.7 \%) \\
157(87.3 \%)\end{array}$ & $\begin{array}{c}64(9.5 \%) \\
227(90.5 \%)\end{array}$ & $\begin{array}{l}190(29.3 \%) \\
482(71.7 \%)\end{array}$ \\
\hline $\begin{array}{l}\text { Marital status } \\
\text { Single } \\
\text { Married } \\
\text { Divorced } \\
\text { Widowed }\end{array}$ & $\begin{array}{c}13(10.4 \%) \\
(83.2 \%) \\
6(4.8 \%) \\
2(1.6 \%)\end{array}$ & $\begin{array}{l}25(9.8 \%) \\
198(78 \%) \\
23(9.1 \%) \\
7(2.8 \%)\end{array}$ & $\begin{array}{c}38(10.0 \%) \\
312(82.3 \%) \\
29(7.7 \%) \\
9(2.4 \%)\end{array}$ & $\begin{array}{c}7(6.3 \%) \\
102(91.1 \%) \\
1(0.9 \%) \\
2(1.8 \%)\end{array}$ & $\begin{array}{c}5(2.8 \%) \\
165(91.2 \%) \\
8(0.71 \%) \\
2(1.1 \%)\end{array}$ & $\begin{array}{c}12(4.1 \%) \\
267(35.4 \%) \\
9(2.0 \%) \\
2(0.7 \%)\end{array}$ & $\begin{array}{c}50(7.4 \%) \\
579(86.2 \%) \\
38(5.7 \%) \\
11(1.6 \%)\end{array}$ \\
\hline $\begin{array}{l}\text { Religion } \\
\text { Mainline } \\
\text { Protestants } \\
\text { Apostolics } \\
\text { Other }\end{array}$ & $\begin{array}{c}15(12 \%) \\
44(35.2 \%) \\
57(45.6 \%) \\
6(4.8 \%)\end{array}$ & $\begin{array}{c}61(24.0 \%) \\
49(19.3 \%) \\
116(45.7 \%) \\
24(9.4 \%)\end{array}$ & $\begin{array}{c}76(20.1 \%) \\
93(12.9 \%) \\
273(72.0 \%) \\
30(8.9 \%)\end{array}$ & $\begin{array}{l}15(13.4 \%) \\
35(31.3 \%) \\
44(39.3 \%) \\
18(16.1 \%)\end{array}$ & $\begin{array}{c}39(21.5 \%) \\
72(39.8 \%) \\
44(24.3 \%) \\
16(8.8 \%)\end{array}$ & $\begin{array}{c}54(18.4 \%) \\
107(36.5 \%) \\
88(30.0 \%) \\
34(11.6 \%)\end{array}$ & $\begin{array}{c}130(19.3 \%) \\
200(29.8 \%) \\
361(53.7 \%) \\
64(9.5 \%)\end{array}$ \\
\hline $\begin{array}{l}\text { Source of Health Education } \\
\text { Village Health Worker (VHW) } \\
\text { Environmental Health Technician } \\
\text { (EHT) } \\
\text { CLINIC } \\
\text { Other }\end{array}$ & $\begin{array}{c}28(22.4 \%) \\
0 \\
92(73.6 \%) \\
0\end{array}$ & $\begin{array}{c}86(33.9 \%) \\
0 \\
155(61.1 \%) \\
0\end{array}$ & $\begin{array}{c}114(32.2 \%) \\
0 \\
247(65.2 \%) \\
0\end{array}$ & $\begin{array}{c}5(7.8 \%) \\
6(9.1 \%) \\
55(83.3 \%) \\
0\end{array}$ & $\begin{array}{c}92(50.8 \%) \\
2(1.1 \%) \\
78(43.1 \%) \\
7(3.9 \%)\end{array}$ & $\begin{array}{c}97(33.1 \%) \\
8(2.7 \%) \\
133(45.4 \%) \\
7(2.4 \%)\end{array}$ & $\begin{array}{c}211(31.4 \%) \\
8(1.2 \%) \\
380(56.5 \%) \\
7(1.2 \%)\end{array}$ \\
\hline
\end{tabular}

Table 1: Socio-demographic characteristics $(n=672)$.

\begin{tabular}{|c|c|c|c|c|c|c|c|}
\hline \multicolumn{4}{|l|}{ Murewa District } & \multicolumn{4}{|c|}{ Seke District } \\
\hline & Macheke & Murewa & Sub-total & Marirangwe & Kunaka & Sub-total & Overall Proportion \\
\hline & $n=125$ & $n=254$ & $n=379$ & $n=112$ & $n=181$ & $n=293$ & $\mathrm{~N}=672$ \\
\hline \multicolumn{8}{|l|}{ Variable } \\
\hline $\begin{array}{l}\text { Received care during pregnancy } \\
95 \% \text { Confidence interval }(\mathrm{Cl})\end{array}$ & $\begin{array}{c}117(93.6 \%) \\
0.89 ; 0.97\end{array}$ & $\begin{array}{c}239(94.1 \%) \\
0.90 ; 0.96\end{array}$ & $\begin{array}{c}357(94.2 \%) \\
0.91 ; 0.97\end{array}$ & $\begin{array}{c}109(97.3 \%) \\
0.92 ; 0.99\end{array}$ & $\begin{array}{c}173(95.6 \%) \\
0.94 ; 0.99\end{array}$ & $\begin{array}{c}283(96.7 \%) \\
0.95 ; 0.99\end{array}$ & $\begin{array}{c}640(95.2 \%) \\
0.93 ; 0.97\end{array}$ \\
\hline $\begin{array}{l}\text { Received care during delivery } \\
95 \% \mathrm{Cl}\end{array}$ & $\begin{array}{l}110(88 \%) \\
0.82 ; 0.93\end{array}$ & $\begin{array}{c}229(90.2 \%) \\
0.86 ; 0.93\end{array}$ & $\begin{array}{c}339(89.4 \%) \\
0.88 ; 0.93\end{array}$ & $\begin{array}{l}94(83.9 \%) \\
0.77 ; 0.90\end{array}$ & $\begin{array}{c}170(93.9 \%) \\
0.91 ; 0.98\end{array}$ & $\begin{array}{c}260(88.7 \%) \\
0.87 ; 0.94\end{array}$ & $\begin{array}{c}599(89.1 \%) \\
0.88 ; 0.92\end{array}$ \\
\hline $\begin{array}{l}\text { Received care } 6 \text { wks. post-delivery } \\
95 \% \mathrm{Cl}\end{array}$ & $\begin{array}{l}112(89.6 \% \\
0.87 ; 0.90\end{array}$ & $\begin{array}{c}243(95.7 \%) \\
0.92 ; 0.98\end{array}$ & $\begin{array}{c}345(91.2 \%) \\
0.89 ; 0.93\end{array}$ & $\begin{array}{c}112(100 \%) \\
0.94 ; 1.00\end{array}$ & $\begin{array}{c}176(97.2 \%) \\
0.94 ; 0.99\end{array}$ & $\begin{array}{c}288(98.3 \%) \\
0.96 ; 0.99\end{array}$ & $\begin{array}{c}633(94.2 \%) \\
0.90 ; 0.93\end{array}$ \\
\hline $\begin{array}{l}\text { First ANC timing } \\
1 \text { st trimester } \\
95 \% \mathrm{Cl} \\
2 \mathrm{nd} \mathrm{trimester} \\
95 \% \mathrm{Cl} \\
3 \mathrm{rd} \mathrm{Trimester} \\
95 \% \mathrm{Cl}\end{array}$ & $\begin{array}{c}19(15.2 \%) \\
0.10 ; 0.23 \\
82(65.6 \%) \\
0.57 ; 0.74 \\
19(15.2 \%) \\
0.10 ; 0.23\end{array}$ & $\begin{array}{c}70(27.6 \%) \\
0.23 ; 0.34 \\
102(40.2 \%) \\
0.34 ; 0.46 \\
71(28.0 \%) \\
0.23 ; 0.34\end{array}$ & $\begin{array}{c}89(23,5 \%) \\
0.18 ; 0.26 \\
184(48.5 \%) \\
0.46 ; 0.50 \\
90(23.7 \%) \\
0.21 ; 0.26\end{array}$ & $\begin{array}{c}49(43.8 \%) \\
0.31 ; 0.49 \\
37(33.0 \%) \\
0.26 ; 0.43 \\
23(20.5 \%) \\
0.16 ; 0.31\end{array}$ & $\begin{array}{c}16(8.8 \%) \\
0.06 ; 0.15 \\
100(55.2 \%) \\
0.50 ; 0.65 \\
58(32.0 \%) \\
0.24 ; 0.38\end{array}$ & $\begin{array}{c}65(22.2 \%) \\
0.18 ; 0.28 \\
137(47.8 \%) \\
0.42 ; 0.53 \\
81(27.6 \%) \\
0.22 ; 0.33\end{array}$ & $\begin{array}{c}154(22.9 \%) \\
0.20 ; 0.26 \\
321(47.8 \%) \\
0.44 ; 0.52 \\
171(25.4 \%) \\
0.22 ; 0.29\end{array}$ \\
\hline $\begin{array}{l}\text { Total ANC visits } \\
\text { One } \\
95 \% \text { Confidence interval } \\
\text { Two } \\
95 \% \mathrm{Cl} \\
\text { Three } \\
95 \% \text { Confidence interval } \\
4 \& \text { above } \\
95 \% \mathrm{Cl}\end{array}$ & $\begin{array}{c}7(5.6 \%) \\
0.03 ; 0.11 \\
4(3.2 \%) \\
0.03 ; 0.05 \\
15(12 \%) \\
0.07 ; 0.19 \\
98(78.4 \%) \\
0.76 ; 0.81\end{array}$ & $\begin{array}{c}7(2.8 \%) \\
0.01 ; 0.06 \\
20(7.9 \%) \\
0.05 ; 0.12 \\
39(15.4 \%) \\
0.11 ; 0.20 \\
187(73.6 \%) \\
0.71 ; 0.76\end{array}$ & $\begin{array}{c}14(3.7 \%) \\
0.02 ; 0.05 \\
24(6.3 \%) \\
0.04 ; 0.08 \\
54(14.2 \%) \\
0.12 ; 0.17 \\
285(75.2 \%) \\
0.74 ; 0.79\end{array}$ & $\begin{array}{c}1(0.9 \%) \\
0.07 ; 0.12 \\
6(5.4 \%) \\
0.04 ; 0.06 \\
16(14.3 \%) \\
0.11 ; 0.15 \\
89(79.5 \%) \\
0.77 ; 0.83\end{array}$ & $\begin{array}{c}10(5.5 \%) \\
0.02 ; 0.10 \\
14(7.7 \%) \\
0.04 ; 0.12 \\
27(14.9 \%) \\
0.12 ; 0.18 \\
127(70.2 \%) \\
0.68 ; 0.73\end{array}$ & $\begin{array}{c}11(3.8 \%) \\
0.02 ; 0.07 \\
20(6.8 \%) \\
0.04 ; 0.11 \\
43(14.7 \%) \\
0.11 ; 0.19 \\
216(73.7 \%) \\
0.68 ; 0.77\end{array}$ & $\begin{array}{c}25(3.7 \%) \\
0.03 ; 0.06 \\
44(6.5 \%) \\
0.05 ; 0.09 \\
68(10.1 \%) \\
0.10 ; 0.12 \\
501(74.6 \%) \\
0.70 ; 0.78\end{array}$ \\
\hline $\begin{array}{l}\text { ANC services received: } \\
\text { Malaria prophylaxis } \\
95 \% \mathrm{Cl} \\
\text { Ferrous } \\
95 \% \mathrm{Cl} \\
\text { Tetanus toxoid } \\
95 \% \mathrm{Cl} \\
\text { Urine Protein test } \\
95 \% \mathrm{Cl}\end{array}$ & $\begin{array}{c}107(85.6 \%) \\
0.79 ; 0.91 \\
115(92 \%) \\
0.90 ; 0.93 \\
107(85.6 \%) \\
0.83 ; 087 \\
19(15.2 \%) \\
0.13 ; 0.17\end{array}$ & $\begin{array}{c}160(63 \%) \\
0.57 ; 0.69 \\
214(84.3 \%) \\
0.82 ; 0.87 \\
181(71.3 \%) \\
0.69 ; 0.73 \\
78(20.6 \%) \\
0.19 ; 0.22\end{array}$ & $\begin{array}{c}267(70.4 \%) \\
0.66 ; 0.75 \\
329(49.0 \%) \\
0.47 ; 0.51 \\
287(75.4 \%) \\
0.70 ; 0.77 \\
97(25.6 \%) \\
0.22 ; 0.27\end{array}$ & $\begin{array}{c}18(16.1 \%) \\
0.13 ; 0.18 \\
107(95.5 \%) \\
0.93 ; 0.97 \\
107(95.5 \%) \\
0.92 ; 0.97 \\
26(23.2 \%) \\
0.20 ; 0.33\end{array}$ & $\begin{array}{c}22(8.7 \%) \\
0.08 ; 0.18 \\
158(62.2 \%) \\
0.61 ; 0.69 \\
156(61.4 \%) \\
0.60 ; 0.65 \\
52(13.7 \%) \\
0.10 ; 0.15\end{array}$ & $\begin{array}{c}40(13.7 \%) \\
0.10 ; 0.18 \\
265(90.4 \%) \\
0.88 ; 0.94 \\
263(89.8 \%) \\
0.88 ; 0.92 \\
78(20.6 \%) \\
0.17 ; 0.22\end{array}$ & $\begin{array}{c}307(45.7 \%) \\
0.42 ; 0.50 \\
594(88.4 \%) \\
0.83 ; 0.90 \\
550(81.8 \%) \\
0.77 ; 0.83 \\
175(26.0 \%) \\
0.22 ; 0.30\end{array}$ \\
\hline
\end{tabular}

Table 2: $\mathrm{MNCH}$ services utilization. 
Citation: Mhlanga M, Zvinavashe M, Gwanzura L, Stray-Pedersen B (2017) Factors Associated with Maternal and Child Health Services Uptake and Their Association with Health Outcomes in Mashonaland East, Zimbabwe. Clinics Mother Child Health 14: 272. doi: 10.4172/20907214.1000272

Page 4 of 7

\begin{tabular}{|c|c|c|c|c|c|c|c|}
\hline \multicolumn{4}{|l|}{ Murewa District } & \multicolumn{4}{|c|}{ Seke District } \\
\hline & Macheke & Murewa & Sub-total & Marirangwe & Kunaka & Sub-total & Overall Proportion \\
\hline & $n=125$ & $n=254$ & $n=379$ & $n=112$ & $n=181$ & $n=293$ & $\mathrm{~N}=672$ \\
\hline \multicolumn{8}{|l|}{ Variable } \\
\hline $\begin{array}{l}\text { Benefits of ANC } \\
2<95 \% \mathrm{Cl}\end{array}$ & $\begin{array}{l}15(12 \%) \\
0.10 ; 0.19\end{array}$ & $\begin{array}{c}53(20.9 \%) \\
0.20 ; 0.28\end{array}$ & $\begin{array}{l}68(17.8 \%) \\
0.18 ; 0.27\end{array}$ & $\begin{array}{c}50(44.6 \%) \\
0.37 ; 0.55\end{array}$ & $\begin{array}{l}93(51.3 \%) \\
0.44 ; 0.60\end{array}$ & $\begin{array}{c}143(48.8 \%) \\
0.46 ; 0.54\end{array}$ & $\begin{array}{c}211(31.4 \%) \\
0.31 ; 0.38\end{array}$ \\
\hline $\begin{array}{l}\text { Knowledge of @ least } \\
2 \text { Danger signs during: } \\
\text { Pregnancy } \\
95 \% \mathrm{Cl}\end{array}$ & $\begin{array}{c}86(68.8 \%) \\
0.64 ; 0.80\end{array}$ & $\begin{array}{c}110(43.3 \%) \\
0.33 ; 0.45\end{array}$ & $\begin{array}{c}196(51.7 \%) \\
0.46 ; 0.56\end{array}$ & $\begin{array}{c}46(41.1 \%) \\
0.32 ; 0.44\end{array}$ & $\begin{array}{c}59(32.6 \%) \\
0.25 ; 0.37\end{array}$ & $\begin{array}{c}105(35.8 \%) \\
0.30 ; 0.36\end{array}$ & $\begin{array}{c}301(44.7 \%) \\
0.40 ; 0.47\end{array}$ \\
\hline $\begin{array}{l}\text { Labour } \\
95 \% \mathrm{Cl}\end{array}$ & $\begin{array}{c}102(81.6 \%) \\
0.72 ; 0.86\end{array}$ & $\begin{array}{c}111(43.7 \%) \\
0.38 ; 0.51\end{array}$ & $\begin{array}{c}213(56.2 \%) \\
0.51 ; 0.61\end{array}$ & $\begin{array}{c}43(38.4 \%) \\
0.36 ; 0.48\end{array}$ & $\begin{array}{l}72(39.8 \%) \\
0.29 ; 0.44\end{array}$ & $\begin{array}{c}115(39.2 \%) \\
0.34 ; 0.43\end{array}$ & $\begin{array}{c}328(48.8 \%) \\
0.44 ; 0.52\end{array}$ \\
\hline $\begin{array}{l}\text { Post-delivery } \\
95 \% \mathrm{Cl}\end{array}$ & $\begin{array}{c}87(69.6 \%) \\
0.64 ; 0.69\end{array}$ & $\begin{array}{c}76(29.9 \%) \\
0.26 ; 0.38\end{array}$ & $\begin{array}{c}124(32.7 \%) \\
0.39 ; 0.49\end{array}$ & $\begin{array}{c}37(33.0 \%) \\
0.27 ; 0.41\end{array}$ & $\begin{array}{l}52(28.7 \%) \\
0.19 ; 0.33\end{array}$ & $\begin{array}{l}89(30.4 \%) \\
0.24 ; 0.34\end{array}$ & $\begin{array}{c}213(31.7 \%) \\
0.34 ; 0.41\end{array}$ \\
\hline $\begin{array}{l}\text { At least } 2 \text { danger signs } \\
\text { to Neonates }\end{array}$ & $\begin{array}{c}66(52.8 \%) \\
0.44 ; 0.61\end{array}$ & $\begin{array}{c}109(42.9 \%) \\
0.37 ; 0.49\end{array}$ & $\begin{array}{c}175(46.2 \%) \\
0.41 ; 0.51\end{array}$ & $\begin{array}{c}49(43.8 \%) \\
0.37 ; 0.46\end{array}$ & $\begin{array}{l}70(38.7 \%) \\
0.29 ; 0.43\end{array}$ & $\begin{array}{c}119(40.6 \%) \\
0.36 ; 0.46\end{array}$ & $\begin{array}{c}294(43.8 \%) \\
0.41 ; 0.48\end{array}$ \\
\hline $\begin{array}{l}\text { Knowledge on the } 3 \\
\text { stages of mother to } \\
\text { child HIV transmission }\end{array}$ & $\begin{array}{c}57(45.6 \%) \\
0.42 ; 0,49\end{array}$ & $\begin{array}{c}93(36.6 \%) \\
0.31 ; 0.41\end{array}$ & $\begin{array}{c}150(39.6 \%) \\
0.37 ; 0.47\end{array}$ & $\begin{array}{l}37(33 \%) \\
0.31 ; 0.48\end{array}$ & $\begin{array}{c}48(26.5 \%) \\
0.20 ; 0.33\end{array}$ & $\begin{array}{c}85(29.0 \%) \\
0.27 ; 0.35\end{array}$ & $\begin{array}{l}235(35 \%) \\
0.33 ; 0.37\end{array}$ \\
\hline
\end{tabular}

Table 3: Knowledge on $\mathrm{MNCH}$.

\begin{tabular}{|c|c|c|c|c|}
\hline Variables & Number of observations & Chi square Value & P-value & Odds Ratio (OR) \\
\hline \multicolumn{5}{|l|}{ Association between: } \\
\hline $\begin{array}{l}\text { Gestational age and birth weight } \\
<37 \text { weeks } \\
37 \text { weeks and above }\end{array}$ & $\begin{array}{c}51(7.6 \%) \\
621(92.4 \%)\end{array}$ & 125 & $0.000^{*}$ & $\begin{array}{c}\mathrm{OR}=2.14 \\
95 \% \mathrm{Cl}: 1.22-3.75)^{*}\end{array}$ \\
\hline $\begin{array}{l}\text { Number of children and maternal complications } \\
<3 \text { children } \\
3+\text { children }\end{array}$ & $\begin{array}{l}370(55.1 \%) \\
302(44.9 \%)\end{array}$ & 340.9 & $0.00^{*}$ & $\begin{array}{c}\text { OR }=4.4 ; \\
95 \% \text { Cl: } 2.45-8.04^{*}\end{array}$ \\
\hline $\begin{array}{l}\text { 1st ANC timing and place of delivery } \\
\text { 1st trimester } \\
\text { 3rd trimester }\end{array}$ & $\begin{array}{l}155(23.1 \%) \\
196(29.2 \%)\end{array}$ & 42.6 & $0.000^{*}$ & $\begin{array}{c}\text { OR=2.84, } \\
95 \% \text { Cl: } 1.53-5.25^{*}\end{array}$ \\
\hline $\begin{array}{l}\text { Decision makers on ANC registration and } 1 \text { st ANC timing } \\
\text { Husband and wife } \\
\text { Wife }\end{array}$ & $\begin{array}{l}400(59.6 \%) \\
220(40.4 \%)\end{array}$ & 50.91 & $0.000^{*}$ & $\begin{array}{c}\text { OR=3.52; } \\
95 \% \text { Cl: } 1.88-6.58^{*}\end{array}$ \\
\hline $\begin{array}{l}\text { Weaning timing and child morbidity } \\
<1.5 \text { years } \\
1.5 \text { years and above }\end{array}$ & $\begin{array}{c}81(36 \%) \\
144(64 \%)\end{array}$ & 648.8 & $0.000^{*}$ & $\begin{array}{c}\text { OR=5.28; } \\
95 \% \text { Cl: } 2.57-9.86^{*}\end{array}$ \\
\hline
\end{tabular}

Table 4: Statistically significant predictors of $\mathrm{MNCH}$ outcomes

nancy, labour and delivery), danger signs to the newborn and knowledge on prevention of mother to child transmission of HIV. Table 3 below summarizes the findings on knowledge.

The study also ascertained the association between socio-demographic factors and morbidity. Association between knowledge on $\mathrm{MNCH}$ and practice was also assessed and so was the association between service utilization and morbidity. Table 4 below summarizes the findings on the statistically significant associations.

The study revealed that there is a statistically significant association between a child's gestational age and birth weight $(\mathrm{OR}=2.14 ; 95 \% \mathrm{CI}$ : 1.22-3.75). Maternal complications were significantly associated with the number of children delivered prior to the last pregnancy $(\mathrm{OR}=4.4$; 95\% CI: 2.45-8.04). First ANC timing was strongly associated with the delivery place in this study $(\mathrm{OR}=2.837,95 \% \mathrm{CI}: 1.53-5.24)$ and so was ANC registration decision ( $\mathrm{OR}=3.52$; 95\% CI: 1.88-6.58). For child health outcomes, the weaning time was significantly associated with child morbidity ( $\mathrm{OR}=5.28$; 95\% CI: 2.57-9.86).

\section{Discussion}

\section{Sociodemographic findings and maternal health outcomes}

The study recruited women $18-49$ years who had children $0-48$ months drawn from two districts in Mashonaland East namely Murehwa and Seke district. The mean age for the women was 28.0 years $(\mathrm{SD}=6.8)$. The majority $437(65.2 \%)$ of the participants had 3 or less children. This aligns well with mean age of the mothers who participated in the study. In Zimbabwe the mean age at first pregnancy is around 20 years and the average child spacing period is two years [4]. $22 \%$ of adolescent females aged 15-19 years in Zimbabwe would have begun childbearing [4]. These facts tally very well with the anticipated number of children for married young women at 28 years. The mean birth weight for children in the study was $3061 \mathrm{~g}(\mathrm{SD}=537)$ and women had a mean weight of $62.5 \mathrm{~kg}(\mathrm{SD}=11.5)$ at first $\mathrm{ANC}$ visit and the mean number of children per woman was $2.6(\mathrm{SD}=1.5)$. $30 \%$ (202) of the participants had not reached the secondary level of education and in terms of marital status 579 (86.2\%) were married. This compares well with the Zimbabwe Demographic health survey report of 2015 that revealed that $27.1 \%$ of the people in Zimbabwe have not reached secondary education level. A study in Nigeria showed that education has a positive association with utilization of Primary Health Care services [5]. With regards to religion, the participants were predominantly Apostolics 361 (53.7\%) followed by Protestants 200 (29.8\%). A study in Egypt on health seeking behavior revealed that socio-cultural resourcefulness is a useful predictor for ANC uptake and facility delivery [6]. 
Citation: Mhlanga M, Zvinavashe M, Gwanzura L, Stray-Pedersen B (2017) Factors Associated with Maternal and Child Health Services Uptake and Their Association with Health Outcomes in Mashonaland East, Zimbabwe. Clinics Mother Child Health 14: 272. doi: 10.4172/20907214.1000272

Page 5 of 7

The study assessed the association between selected socio-demographic variables with maternal and child health outcomes. The variables that were selected were maternal age, gestational age at delivery, number of children, sex of household head, and religion of the participant and literacy levels. The results revealed that there were no statistically significant associations between maternal age and maternal complications $\left(\chi^{2}=132 ; \mathrm{p}<0.458\right)$; maternal age and mode of delivery $\left(\chi^{2}=136 ; \mathrm{p}<0.971\right)$ and sex of household head and place of delivery $\left(\chi^{2}=10.04 ; p<1.000\right)$. These results slightly differ with what Okwaraji and others found in Ethiopia where educated women lived closer to health centers than uneducated women (Adjusted mean difference travel time $-41 \min 95 \%$ CI -50 ;-31). Women aged $15-20$ years were more likely to live in poor access areas compared with women aged 21-30 years (adjusted mean difference travel -11 min 95\% CI -23-0) [3]. One would expect the association between maternal age and complications to be significant since there is biological plausibility to that effect. However, the contrasting results could be explained in terms of the mean age (28 years) for the participants in this study. It is less likely that these young mothers would report age related maternal complications.

However, the study revealed a statistically significant association between gestational age at delivery and birth weight $(\mathrm{OR}=2.14 ; 95 \% \mathrm{CI}$ : 1.22-3.75).), and also between number of children and maternal complications $(\mathrm{OR}=4.4 ; 95 \% \mathrm{CI}: 2.45-8.04)$. This meant that children born with a gestational age of less than 37 weeks were 2.14 times more likely to be low-birth weight babies when compared with those born after 37 weeks. Similarly, mothers with more than three children were 4.4 times more likely to have maternal complications when compared with those with less than 3 children. These findings confirm conclusions that have been reached in similar studies. However, a study in the US failed to explain the decline in fetal growth among U.S., term, singleton neonates by trends in maternal and neonatal characteristics, changes in obstetric practices, or concurrent decreases in gestational length [7].

\section{MNCH practices and maternal health outcomes}

Overall, 639 (95.2\%) of the participants reported having received health care services at least once during their last pregnancy, 599 (89.1\%) during delivery and 66 (94.2\%) post-delivery. A similar study in Egypt reported only $58.5 \%$ of mothers accessing ANC services before delivery [6]. Zimbabwe is one of the countries in Africa that are doing well in terms of promotion of access of $\mathrm{MNCH}$ services during pregnancy. Only 154 (22.9\%) of the participants registered for ANC in the first trimester while $321(47.7 \%)$ and 171 (25.4\%) booking in the second and third trimester respectively. This poses great risk to both the mother and the unborn baby since there are fixed window periods under which some risks such as risk of transmission of HIV from the mother to the unborn child can be averted. Reasons for not accessing care services along the continuum of care were explored. Religious beliefs were cited as the reason for not accessing care by $10(23.2 \%)$ of those who did not seek care during pregnancy. Of the 43 who did not access any service during pregnancy, $6(14 \%)$ said the health center is too far and $9(20.9 \%)$ cited financial constraints for user fees. A study in India revealed that household income is a determinant of institutional delivery (OR: 2.74 95\% CI 1.81, 4.15) [8]. Reasons cited for failure to access services during delivery included distance to the clinic which then resulted in emergencies before arrival 26 (60.5\%); religious beliefs and financial constraints [8].

Utilization of postnatal services at 6 weeks was generally high in this study $633(94.2 \%)$ yet very low (15\%) within the first 72 h postdelivery. This could be explained by the fact that in Zimbabwe, postnatal care (PNC) and child health care services are now being integrated at 6 weeks. The uptake of PNC services at 6 weeks went up as mothers will be bringing their children for immunization hence take the same opportunity to get PNC services. PNC uptake within $72 \mathrm{~h}$ remains a tall order since most health facilities do not have enough infrastructures to keep the mothers post-delivery for the recommended 3 days. Most women find it unnecessary to report back at the health facility on day 3 due to various reasons including distance to the clinic, a feeling of wellness and general belief that new-born babies must be kept in-doors for at least one month. A study in Indonesia found out that the prevalence of non-attendance for post-natal care services was consistently higher in rural areas than urban areas. Maternal factors associated with lack of PNC included low household wealth index, low education levels, lack of knowledge of pregnancy-related complications and distance to the health facility [9].

The study revealed that $4.9 \%$ of the 672 participants had confirmed proteinuria and the prevalence was comparable in both the control and intervention arm participants, $17.9 \%$ and $19.6 \%$ respectively for those participants who were screened for proteinuria. These results revealed a glaring gap in terms of screening pregnant women for proteinuria. This increases the risk of late detection and management of pre-eclampsia. Screening pregnant women for proteinuria should be a routine practice in Zimbabwe. This study also revealed that pre-eclampsia had a higher prevalence than any other maternal complication during pregnancy which justifies the need to prioritize the urine test for proteinuria in all health centres.

The study also checked the association between variables on access and utilization of $\mathrm{MNCH}$ services and maternal and child health outcomes. These variables were inclusive of religion, literacy levels; first ANC timing and HIV status. There was no significant association between religion and timing for first ANC $\left(\chi^{2}=18.6 ; \mathrm{p}<0.820\right)$; literacy level and timing for first ANC $\left(\chi^{2}=18.6 ; p<0.330\right)$; receiving care along the continuum and maternal complications $\left(\chi^{2}=7.75 ; \mathrm{p}<0.560\right)$ and between HIV status and maternal complications $\left(\chi^{2}=17.8 ; p<1.000\right)$. A similar study in Ethiopia revealed that women's educational status, distance to the nearest health facility and access to maternal and child care information were predictors of institutional child birth service utilization [10]. Safe motherhood education using communication networks in rural and urban communities is critical.

However, the study revealed statistically significant associations between first ANC timing and place of delivery (OR=2.84, 95\% CI: 1.53 5.25); decision makers on ANC registration and timing for first ANC (OR=3.52; 95\% CI: 1.88-6.58) and also between weaning timing and child morbidity (OR=5.28; 95\% CI: 2.57-9.86). Mothers who booked early for ANC were 2.84 times more likely to deliver at a health facility under the care of a professional health service provider. Similarly, most of the decision makers on ANC registration were husbands and wives (58\%) followed by women $(29.2 \%)$ and then men (12.8\%). There was higher likelihood of early ANC booking amongst mothers who could make independent decisions on maternal and child health and those families who jointly made decisions on $\mathrm{MNCH}$ yet there was less likelihood on the same in highly patriarchal families where the husband was the pivot for all decision made on health and other facets of life. A study by Banke et al. revealed that use of ANC services predicted use of skilled birth attendance and use of both predicted use of PNC services [6]. A study in Nepal revealed that men with higher household income were more likely (AOR: 5.91, 95\% CI 4.02, 8.70) to have ANC with their wives than man with no education or with primary level education [11]. Children who were weaned earlier than the recommended minimum of 24 months were likelier to be sick as compared to those whose mothers' breastfeed for at least 24 months with age appropriate complementary feeding [11]. 
Citation: Mhlanga M, Zvinavashe M, Gwanzura L, Stray-Pedersen B (2017) Factors Associated with Maternal and Child Health Services Uptake and Their Association with Health Outcomes in Mashonaland East, Zimbabwe. Clinics Mother Child Health 14: 272. doi: 10.4172/20907214.1000272

Page 6 of 7

\section{MNCH knowledge of mothers and health outcomes/practices}

In this study, various knowledge aspects on maternal and child health care were assessed. High knowledge is usually associated with better health practices and ultimately better health outcomes for mothers and their children. In this study mothers' knowledge on the benefits of ANC was assessed. Participants were also assessed on knowledge on danger signs along the continuum of care and ways of managing the danger signs in both mothers and neonates. The study also assessed the mothers' knowledge on possible stages at which HIV can be transmitted to a baby since HIV and AIDS is prevalent in sub -Saharan Africa.

Only 211 (31.5\%) of the participants knew at least two benefits of Antenatal Care out of the possible five benefits that the study had prioritized. Antenatal care is critical for the maintenance of the health of the mother prior to delivery and is crucial for assessing possible risks to the mother and the unborn baby. During antenatal care, the growth and development of an unborn baby is assessed and mothers also get useful health information with regards to their pregnancy. Life-saving services such as prevention of mother to child transmission of HIV (PMTCT) services are offered and so are the prophylactic services to curb risk for maternal and new-born complications. It is then worrisome that more than half of the mothers could not come up with at least two benefits of ANC. Such a deficit in knowledge could later on translate to devaluation of recommended behaviors that are meant to save lives and increase chances of morbidity and mortality. This could also be the reason why most of the participants were booking in the second and third trimester.

On danger signs to the mother during pregnancy, only 301 (44.7\%) of the participants knew at least two danger signs out of a possible seven that the study prioritized. Danger signs during pregnancy include vaginal bleeding; pre-term labour; severe abdominal pain; severe headache; loss of consciousness; swelling and severe weakness. The low levels of knowledge amongst the majority of the interviewed women could also partially explain why most of them book late for ANC.

Danger signs during delivery include eclampsia; severe vaginal bleeding; sepsis; severe weakness; shortness of breath; prolonged labour; swelling; high fever; foul vaginal discharge; severe headache/blurred vision; convulsions and retained placenta. Of these danger signs, only 328 (48.8\%) of the participants knew at least two and the commonest known danger sign was vaginal bleeding. Only 89 (30.4\%) of the participants knew at least two danger signs post-delivery and this could be the reason why most mothers don't report for postnatal care within the recommend $72 \mathrm{~h}$ especially when they think they delivered well and they have nothing to worry about. A similar study in Uganda found out that 52\% of women knew at least one key danger sign during pregnancy, $72 \%$ during delivery and $72 \%$ during postpartum and only $19 \%$ had knowledge of 3 or more key danger signs during the three periods [12]. Some of these women later on present with advanced maternal complications or present with neonates who have severe complications.

Only $294(43.8 \%)$ of the participants knew at least two neonatal danger signs out of possible 8 danger signs that the study was assessing. The eight neonatal danger signs were difficult breathing, chest in drawing, too hot or too cold, red cord stump or cord with pus, eyes filled with pus, jaundice and blue extremities. Knowledge on danger signs to the new-born is very essential for child survival. A study in Ethiopia showed that mothers who had knowledge of three or more neonatal danger signs (good knowledge) were found to be $18.2 \%$ (95\% CI 15.1,21.3\%) [13]. The odds of having good knowledge was positively associated with mother's (AOR $=3.41,95 \% \mathrm{CI} 1.37,8.52)$ and father's
(AOR $=3.91,95 \%$ CI 1.23, 12.36) higher educational achievement. Similarly, the odds of having good knowledge about neonatal danger signs were higher among Antenatal care $(\mathrm{AOR}=2.28,95 \% \mathrm{CI} 1.05,4.95)$ and Postnatal care attendant mothers ( $\mathrm{AOR}=2.08,95 \% \mathrm{CI} 1.22$, 3.54). Furthermore, access to television was also associated with mothers' good knowledge about neonatal danger signs ( $\mathrm{AOR}=3.49,95 \% \mathrm{CI} 1.30,9.39$ ) [13]. If new-borns are left unobserved for these signs, chances of dying within the first 7 days (early neonatal period) are very high hence the need for all mothers to be knowledgeable on these signs. One can argue that to some extent, this could be the reason why neonatal mortality rate is high in sub-Saharan Africa.

Elimination of Mother to Child Transmission of HIV is at the center of developmental goals globally. At this stage of fighting HIV and AIDS, one would expect mothers to be knowledgeable on the ways in which HIV can be transmitted from a mother to a born or unborn child. To this effect, the participants' knowledge on the stages at which a mother can transmit HIV to their child was evaluated. Two hundred and thirty five $(35 \%)$ of the mothers interviewed knew that HIV can be transmitted at all the anticipated three stages which are during pregnancy, during delivery and during the breastfeeding stage. More than $60 \%$ of the participants knew at least two stages. This result shows that there is still a knowledge gap amongst mothers which can also influence their choices and practices in PMTCT. A similar study conducted in Nigeria revealed that $90 \%$ of the respondents were aware that HIV/AIDS can coexist with pregnancy, but only $68 \%$ were aware of mother-to-child transmission. Trans-placental route, vaginal delivery and breastfeeding were identified as routes of transmission from mother to child by $65 \%$, $38 \%$ and $52 \%$ of respondents, respectively [14]. Similarly, in a study in Mexico Less than one-third (30\%) knew that HIV could be transmitted to a child during delivery, and $36 \%$ knew that HIV could be transmitted by breast-feeding. Only $27 \%$ knew that an MTCT could be prevented. Prenatal patients were more likely to know that MTCT was preventable (prenatal: $31 \% v s . \mathrm{L} \& \mathrm{D} 25 \% ; \mathrm{P}=0.02$ ). Logistic regression indicated that prenatal patients (odds ratio $=1.49$, confidence interval 1.07-2.07) were more likely to know that HIV could be transmitted through breastfeeding [15].

An analysis was made to evaluate the association between knowledge and maternal health practices/outcomes. There were no statistically significant associations between knowledge of danger signs during pregnancy and timing for first ANC $\left(\chi^{2}=278.3 ; \mathrm{p}<1.000\right)$; knowledge of ANC benefits and timing for first ANC $\left(\chi^{2}=119.2 ; p<0.897\right)$; knowledge of delivery danger signs and place of delivery $\left(\chi^{2}=172.7 ; p<1,000\right)$ and also between knowledge of ANC benefits and number of ANC visits $\left(\chi^{2}=155 ; p<0.170\right)$. These results could partially be explained by the fact that most of the participants' knowledge was generally low.

On the contrary, a study in Uganda sought to explore the association between knowledge of obstetric danger signs and birth preparedness among recently delivered revealed a statistically significant relationships between knowledge of at least one key danger sign during pregnancy or during postpartum and birth preparedness (OR 1.8, 95\% CI: 1.2-2.6) and (OR 1.9, 95\% CI: 1.2-3.0) respectively [12].

\section{Conclusion}

This study revealed that though there is a satisfactory health seeking behaviour amongst pregnant and lactating women, there is still a significant gap in knowledge of critical recommended MNCH practices. Both good knowledge and good practices are essential in the reduction of preventable maternal and child morbidity and mortality. Universal maternal health access is only achievable if the community and women make deci- 
Citation: Mhlanga M, Zvinavashe M, Gwanzura L, Stray-Pedersen B (2017) Factors Associated with Maternal and Child Health Services Uptake and Their Association with Health Outcomes in Mashonaland East, Zimbabwe. Clinics Mother Child Health 14: 272. doi: 10.4172/20907214.1000272

Page 7 of 7

sions about their own health in a supportive environment. There is need to have community based interventions for maternal health access. The study results showed that the quality and methods of delivery of antenatal care education should be reviewed to improve effectiveness.

\section{References}

1. Thaddeus S, Maine D (1994) Too far to walk: maternal mortality in context. Soc Sci Med 38: 1091-1110.

2. Zimbabwe Peri-natal audit report 2007.

3. Okwaraji YB, Webb EL, Edmond KM (2015) Barriers in physical access to maternal health services in rural Ethiopia. BMC Health Serv Res 15: 493.

4. Zimbabwe Demographic Health Survey Report 2015.

5. Sule SS, ljadunola KT, Onayade AA, Fatusi AO, Soetan RO, et al. (2008) Utilization of primary health care facilities: lessons from a rural community in southwest Nigeria. Niger J Med 17: 98-106.

6. Benova L, Campbell OM, Sholkamy H, Ploubidis GB, et al. (2014) Socioeconomic factors associated with maternal health-seeking behaviours among women from poor households in rural Egypt. Int J Equity Health 13: 111

7. Donahue SM, Kleinman KP, Gillman MW, Oken E (2010) Trends in birth weight and gestational length among singleton term births in the United States: Obstet Gynecol 115: 357-364.

8. Singh L, Rai RK, Singh PK (2012) Assessing the utilization of maternal and child health care among married adolescent women: evidence from India. J Biosoc Sci 44: 1-26.

9. Titaley CR, Dibley MJ, Roberts CL (2009) Factors associated with nonutilization of postnatal care services in Indonesia. J Epidemiol Community Health 63: 827-831.

10. Hailu D, Berhe H (2014) Determinants of institutional childbirth service utilization among women of childbearing age in urban and rural areas of Tsegedie district, Ethiopia. Midwifery 30: 1109-1117.

11. Banke-Thomas OE, Banke-Thomas AO, Ameh CA (2017) Factors influencing utilization of maternal health services by adolescent mothers in Low-and middleincome countries: a systematic review. BMC Pregnancy and Childbirth 17: 65.

12. Kabakyenga JK, Östergren PO, Turyakira E, Pettersson KO (2011) Knowledge of obstetric danger signs and birth preparedness practices among women in rural Uganda. Reprod Health 8: 33.

13. Nigatu SG, Worku AG, Dadi AF (2015) Level of mother's knowledge about neonatal danger signs and associated factors in North West of Ethiopia: a community based study. BMC Res Notes 8: 309.

14. Abiodun MO, ljaiya MA, Aboyeji PA (2007) Awareness and knowledge of mother-to-child transmission of HIV among pregnant women. J Natl Med Assoc 99:758-763.

15. Becka CM, Chacón-Cruz E, Araneta MR, Viani RM (2015) Lack of knowledge about mother-to-child HIV transmission prevention in pregnant women at Tijuana General Hospital, Baja California, Mexico. J Int Assoc Provid AIDS Care 14: 72-76. 